assignment of a diagnostic score in clinical practice can be useful to apply early environmental and dietary prevention measures.

770

\section{THE STATE OF CYTOKINE LINKS OF IMMUNITY AT HEPATOGENIC ULCERS AND WAYS OF CORRECTION}

O.M. Horlenko', O.M. Moskal ${ }^{1}$, E.I. Arshiy², F.V. Horlenko ${ }^{3}$, B.M. Halay ${ }^{2}$

${ }^{1}$ Pediatrics with Infectious Diseases, ${ }^{2}$ Internal Medicine, ${ }^{3}$ Surgical Diseases, Uzhgorod National University, Medical Faculty, Uzhgorod, Ukraine

Background and aims: The great number of researches is confirmed, that development of ulcerous illness and chronic gastritis is accompanied violations of cytokine station. They present are given about the change of products of proinflammatory and anti-inflammatory interleukins in the mucus shell of stomach and duodenum.

Methods: We study the levels of IL-1ß, IL-2, IL-4, IL-8 in the serum blood of patients with hepatogenic ulcers and erosions which complicated the chronic diffuse diseases of liver (CHDDL). We observed 120 patients, amonge them 72 patients with the cirrhosis of liver (CL) and 48 patients by chronic active hepatitis (CHAH) by alcoholic etiology. The I-st group of patients used the base treatment at CL with including - "Ursofalk" and "Dufalac", 1 month, II-nd group used the base treatment.

Result: In I-st group of patients levels of IL-1ß, IL-4 under used of treatment decreased to the reference dates. In the II-nd group of patients the levels of all of interleukins after treatment remained higher normal indexes. There were folloving reliable differential levels of interleukins of I-st and II-nd groups after treatment: concentration of IL-1ß $(p<0,001)$, IL-4 $(p<0,01)$.The indexes of interleukins in the serum blood of patients of the II group remain higher than indexes of I of group.

Conclusions: After used complex treatment concentration of $\mathrm{IL}-1 \beta$ decreased in 2 times comparatively with an initial level $(p<0,01)$, concentration of IL-2 in 1,7 times $(p<0,05)$, concentration of IL-4 in 1,2 times $(p>0,05)$, concentration of IL-8 in 1,4 times $(p>0,05)$.

\section{THE MANAGEMENT OF ACUTE GASTROENTERITIS IN CHILDREN IN PORTUGAL}

H. Antunes ${ }^{1,2}$, L. Januário ${ }^{3}$, H. Flores ${ }^{4}$, J. Cabral ${ }^{4}$, F. Santos ${ }^{4}$, I. Pó ${ }^{4}$, A.I. Lopes ${ }^{5}$

${ }^{1}$ Life and Health Sciences Research Institute (ICVS), '2Gastroenterology, Hepatology and Nutrition Unit, Pediatric Dpt. on behalf of the Gastroenterology Section of the Paediatric Portuguese Society (SPP) and on behalf of the $S P P$, Braga Hospital, Braga, ${ }^{3}$ Pediatric Dp. on behalf of the SPP, Pediatric Hospital, Coimbra, ${ }^{4}$ Pediatric Dpt. on behalf of the Gastroenterology Section of the SPP, Estefânia Hospital, ${ }^{5}$ Pediatric Dpt. on behalf of the Gastroenterology Section of the SPP, S. Maria Hospital, Lisboa, Portugal

Aim: To evaluate the knowledge/practice in the AGE's management in children.

Methods: An anonymous semi-structured questionnaire(21 questions) was applied to paediatricians, paediatrician fellows(PF) and general practitioners(GP) from July 7 to September 25,2009 , in the site of SPP. The Zprobe was used to compare paediatricians/PF(1);paediatricians/ $\mathrm{GP}(2)$.

Results: There were 430 answers:paediatricians $261(27.3 \%$ registered paediatricians in the national Board), PF 94, GP 83. They answered they knew ESPGHAN'recommendations for Oral Rehydration Solution(ORS) use: paediatricians 82.0\%;PF $\quad 76.6 \%$;GP $\quad 41.0 \%,(1) p=0.220 ;(2) p<$ 0.001 . The sodium recommended ORS content was correctly answered by paediatricians:68.6\%; PF:69.1\%; GP:32.5\%,(1)p=0.397;(2)p< 0.001 . ORS was reported to be used in $86.3 \%$ of cases (paediatricians:88.5\%; PF:95.7\%;GP:68.7\%,(1) $\mathrm{p}=0.017 ;(2) \mathrm{p}<0.001) ;$ and home made "ORS" in $\quad 12.8 \% \quad$ (GP:"yes":25.3\%;PF:"no":57.4\% $,(2) p<0.001)$. Nineteen percent answered they would ever recommend stopping diet. The delay until reassuming normal diet was respectively:< 4 hours(h)54.0\%(normal diet interval); 4-6h:38.7\%; >6h:7.9\%. Maintenance of breastfeeding was reported in $96.3 \%$; the use of lactose-free milk or diluted milk was not recommend in 53.0\%(paediatricians:53.4\%; PF:69.1\%; GP:33.7\%,(1)p=0.008;(2)p< 0.001) and $86.5 \%$ (pediatricians:85.8\%; PF: $94.7 \%$; GP:79.5\%,(1)p=0.008;(2)p=0.176), respectively. The recommendation to modify the diet was 